31 July 1998

\title{
OCTONIONIC MÖBIUS TRANSFORMATIONS
}

\author{
Corinne A. Manogue \\ Department of Physics, Oregon State University, Corvallis, OR 97331, USA \\ corinne@physics.orst.edu \\ Tevian Dray \\ Department of Mathematics, Oregon State University, Corvallis, OR 97331, USA \\ tevian@math.orst.edu
}

\begin{abstract}
A vexing problem involving nonassociativity is resolved, allowing a generalization of the usual complex Möbius transformations to the octonions. This is accomplished by relating the octonionic Möbius transformations to the Lorentz group in 10 spacetime dimensions. The result will be of particular interest to physicists working with lightlike objects in 10 dimensions.
\end{abstract}

\section{INTRODUCTION}

It is well-known that the Möbius transformations generate the conformal group in the plane, and that they can be identified using stereographic projection with conformal transformations on the sphere. As emphasized so elegantly by Penrose [1] in his twistor program, this identification allows the Möbius transformations to be identified with the Lorentz group $\mathrm{SO}(3,1)$ in 4 dimensions, since each Lorentz transformation induces a conformal transformation on the 2-sphere of null directions.

In this paper we generalize all of this structure in a natural way to the octonions. A key piece of the puzzle is the use of the octonionic Lorentz transformations in 10 dimensions as given by Manogue \& Schray [2]. We find that, despite the apparent obstacles due to nonassociativity, the identification of octonionic Möbius transformations with $\mathrm{SO}(9,1)$, is straightforward.

After much of this work was completed, we discovered the earlier work of Dündarer, Gürsey \& Tze [3,4], who discuss conformal transformations of $\mathcal{R}^{8}$ using similar techniques. As discussed in more detail below, our treatment differs from theirs in a way which could have important consequences for the study of lightlike objects in 10 dimensions. We also point out an error in their treatment of $G_{2}$, which invalidates the precise form of the Möbius representation given in [3,4]; this is easily corrected.

In order to keep the article self-contained, we review our basic ingredients in the first two sections: octonions in Section 2, and complex Möbius transformations in Section 3. These sections can be safely omitted by the knowledgeable reader. In Section 4 we describe the results of Manogue \& Schray [2], which show how to resolve the associativity difficulties inherent in defining finite octonionic Lorentz transformations. These same ideas are then used in Section 5 to obtain the new result of this paper, namely the generalization of 


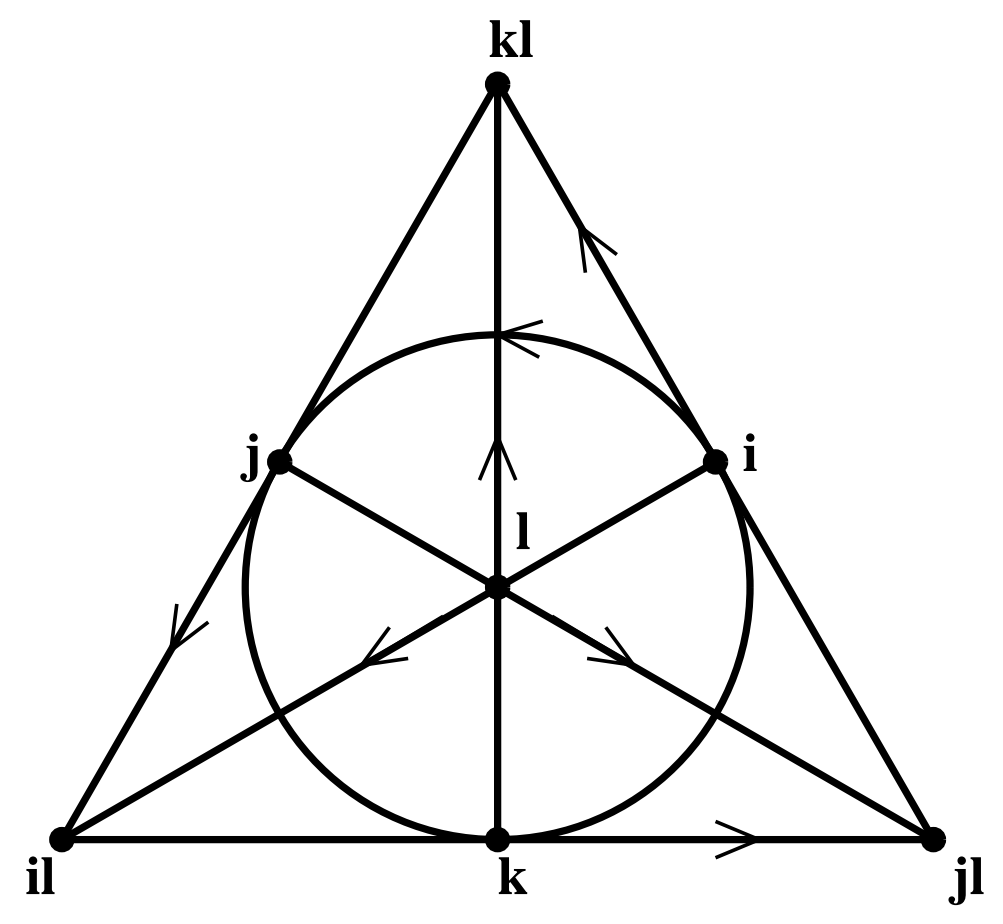

Figure 1: The representation of the octonionic multiplication table using the 7point projective plane, where we have used the conventional names $\{i, j, k, k l, j l, i l, l\}$ for $\left\{e_{2}, \ldots, e_{8}\right\}$. Each of the 7 oriented lines gives a quaternionic triple.

Möbius transformations from the complexes to the octonions. In Section 6 we discuss the work of Dündarer, Gürsey and Tze, both discussing how our approach differs from theirs and correcting the aforementioned error. Finally, we discuss our results in Section 7.

\section{OCTONIONS}

The octonions $\mathcal{O}$ are the nonassociative, noncommutative, normed division algebra over the reals. In terms of a natural basis, an octonion $a$ can be written

$$
a=\sum_{q=1}^{8} a^{q} e_{q}
$$

where the coefficients $a^{q}$ are real, and where the basis vectors satisfy $e_{1}=1$ and

$$
e_{q}^{2}=-1 \quad(q=2, \ldots, 8)
$$

We refer to the latter as imaginary basis units; they anticommute

$$
e_{q} e_{r}=-e_{r} e_{q} \quad(q \neq r ; q, r=2, \ldots, 8)
$$

and products of two different imaginary basis units yield a third, i.e. $e_{q} e_{r}= \pm e_{s}$ for some $s$. The full multiplication table is conveniently encoded in the 7-point projective plane, shown in Figure 1. The product of any two imaginary units is given by the third unit on the unique line connecting them, with the sign determined by the relative orientation. 
The associator of three octonions is

$$
[a, b, c]=(a b) c-a(b c)
$$

which is totally antisymmetric in its arguments and has no real part. Although the associator does not vanish in general, the octonions do satisfy a weak form of associativity known as alternativity, namely

$$
[a, b, a]=0
$$

The underlying reason for this is that two octonions determine a quaternionic subalgebra of $\mathcal{O}$, so that any product containing only two octonionic directions is associative.

Octonionic conjugation is given by reversing the sign of the imaginary basis units, so, with $a$ as above,

$$
\bar{a}=a^{1} e_{1}-\sum_{q=2}^{8} a^{q} e_{q}
$$

Conjugation is an antiautomorphism, since it satisfies

$$
\overline{a b}=\bar{b} \bar{a}
$$

The inner product on $\mathcal{O}$ is the one inherited from $\mathcal{R}^{8}$, namely

$$
\langle a, b\rangle=\sum_{q} a^{q} b^{q}
$$

which can be rewritten as

$$
\langle a, b\rangle=\frac{1}{2}(a \bar{b}+b \bar{a})=\frac{1}{2}(\bar{b} a+\bar{a} b)
$$

Finally, the norm of an octonion is just

$$
|a|=\sqrt{a \bar{a}}=\sqrt{\langle a, a\rangle}
$$

which satisfies the defining property of a normed division algebra, namely

$$
|a b|=|a||b|
$$

\section{COMPLEX MÖBIUS TRANSFORMATIONS}

The unit sphere $\mathcal{S}^{2} \subset \mathcal{R}^{3}$ is related to the Riemann sphere (the complex plane with a point at infinity added) via stereographic projection from the north pole, which takes the point $(x, y, z)$, with $x^{2}+y^{2}+z^{2}=1$, to the point

$$
w=\frac{x+i y}{1-z}=\frac{1+z}{x-i y}
$$


Under this transformation, the north pole is mapped to the point at infinity.

As discussed in detail by Penrose and Rindler [1], we can regard $\mathcal{S}^{2}$ as the set of future (or past) null directions, specifically as the intersection of the future light cone of the origin in 4-dimensional Minkowski space with the hypersurface $t=1$. Other points on a given null ray are obtained by scaling with $t$, and we can extend stereographic projection to a map on the entire light cone via

$$
w=\frac{x+i y}{t-z}=\frac{t+z}{x-i y}
$$

with the condition $x^{2}+y^{2}+z^{2}=t^{2}$. Penrose and Rindler show how to obtain this correspondence directly by an orthogonal projection in Minkowski space, rather than via stereographic projection.

We can further identify $\mathcal{S}^{2}$ with the complex projective space $\mathcal{C P}^{1}$, the space of complex lines in $\mathcal{C}^{2}$, which is given by

$$
\mathcal{C P}^{1}=\left\{[(b, c)] \in \mathcal{C}^{2}:(b, c) \sim(\xi b, \xi c) \quad \forall 0 \neq \xi \in \mathcal{C}\right\}
$$

where the square brackets denote equivalence classes under the equivalence relation $\sim$. Then each $[(b, c)] \in \mathcal{C P}^{1}$ can be identified with the point $w$ in the complex plane given by

$$
w=\frac{b}{c}
$$

which is further identified with a point in $\mathcal{S}^{2}$ via $(11) ;[(b, 0)]$ is to be identified with the north pole, corresponding to $w=\infty$. Stereographic projection (11) can be thought of as a special case of (14) with $b$ or $c$ real.

The Möbius transformations in the complex plane are the complex mappings of the form ${ }^{1}$

$$
w \mapsto \frac{\alpha w+\beta}{\gamma w+\delta}
$$

where $\alpha \delta-\beta \gamma \neq 0$. It is usually assumed without loss of generality that the complex numbers $\alpha, \beta, \gamma, \delta$ satisfy

$$
\alpha \delta-\beta \gamma=1
$$

Möbius transformations are the most general analytic transformation of the Riemann sphere to itself. Using (14), we can rewrite (15) as

$$
\frac{b}{c} \mapsto \frac{\alpha b+\beta c}{\gamma b+\delta c}
$$

The Möbius transformation (17) does not depend on the particular choice of $b$ and $c$ in the equivalence class $[(b, c)]$, which allows us to view it as acting on $\mathcal{C P}^{1}$.

1 An excellent description of these transformations, and their relation to Lorentz transformations, appears in $\S 1.2$ and $\S 1.3$ of [1]. 
Now consider a Hermitian $2 \times 2$ matrix $A$, which we can write as

$$
A=\left(\begin{array}{cc}
t+z & x+i y \\
x-i y & t-z
\end{array}\right)
$$

We can identify $A$ with the Minkowski vector $A^{\mu}=(t, x, y, z)$. Furthermore, the norm of $A^{\mu}$ is given by

$$
A^{\mu} A_{\mu}=-\operatorname{det} A
$$

where our signature is $(-+++)$. In particular, $A$ corresponds to a null direction if and only if $\operatorname{det} A=0$, and in this case we can always write

$$
A=v v^{\dagger}
$$

where

$$
v=\left(\begin{array}{l}
b \\
c
\end{array}\right)
$$

is a spinor. Conversely, the matrix square of any spinor $v$ corresponds to a null vector. But the space of null directions is precisely $\mathcal{S}^{2}$, as can be seen by simply identifying $\{\xi v\}$ with $w=b / c$, noting that the overall scale is irrelevant. (The remaining phase freedom in $b$ and $c$ corresponds to the Hopf fibration.)

Using these various identifications, we can rewrite a Möbius transformation (15) as a map on spinors

$$
v \mapsto M v
$$

with $v$ as above and where

$$
M=\left(\begin{array}{ll}
\alpha & \beta \\
\gamma & \delta
\end{array}\right)
$$

Imposing the condition (16), we see that $\operatorname{det} M=1$, so that $M$ is (the spinor representation of) a Lorentz transformation. As could be expected from (20), $M$ acts on vectors $A$ via

$$
A \mapsto M A M^{\dagger}
$$

which preserves the determinant (i.e. the norm) of $A$ as required.

We thus see that Möbius transformations are exactly the same as Lorentz transformations. Note the key role played by associativity, which allows one to multiply numerator and denominator of a Möbius transformation by $c$, thus permitting a reinterpretation as a matrix equation.

\section{LORENTZ TRANSFORMATIONS}

Manogue \& Schray [2] give an explicit representation of a set of generators for finite Lorentz transformations in 10 spacetime dimensions. Their results can be summarized as follows.

Let $A$ be a Hermitian $2 \times 2$ octonionic matrix, so that we can write

$$
A=\left(\begin{array}{cc}
p & a \\
\bar{a} & m
\end{array}\right)
$$


where $p, m$ are real, and where $a$ is an arbitrary octonion. Just as in the complex case, we can associate $A$ with a (real) vector $A^{\mu}=\left(a^{0}, \ldots, a^{9}\right)$ in (10-dimensional) Minkowski space via

$$
\begin{aligned}
p & =a^{0}+a^{9} \\
m & =a^{0}-a^{9} \\
a & =\sum_{q=1}^{8} a^{q} e_{q}
\end{aligned}
$$

As in the complex case, the norm of $A^{\mu}$ is given by (19); the determinant is well-defined since $A$ is Hermitian. If $A$ is null we still have (20) and (21); the freedom in choosing $b$ and $c$ again corresponds to a real scale and the Hopf fibration (of $\mathcal{S}^{15}$ in this case).

A Lorentz transformation $M$ acts on $A$ via (24), and leaves the determinant invariant, thus preserving the norm of $A^{\mu}$. The first requirement on $M$ is that this be well-defined, i.e. that

$$
(M A) M^{\dagger}=M\left(A M^{\dagger}\right)=: M A M^{\dagger}
$$

In particular, this means that $M A M^{\dagger}$ is indeed Hermitian. Manogue \& Schray note that for this to be the case, either $M$ must be complex, i.e. the components of $M$ lie in a complex subspace of $\mathcal{O}$, or the columns of the imaginary part of $M$ must be real multiples of each other. In either case,

$$
\operatorname{det}\left(M A M^{\dagger}\right)=\operatorname{det}\left(M M^{\dagger}\right) \operatorname{det} A
$$

so that a further condition for $M$ to be a Lorentz transformation is

$$
\operatorname{det}\left(M M^{\dagger}\right)=1
$$

It is well-known, however, that not all Lorentz transformations in 10 dimensions can be written in the form (24). Because of the lack of associativity, not all Lorentz transformations can be achieved with a single matrix $M$. Manogue \& Schray [2] show, however, that nested transformations are sufficient, e.g.

$$
A \mapsto M_{n}\left(\ldots\left(M_{1} A M_{1}^{\dagger}\right) \ldots\right) M_{n}^{\dagger}
$$

The generating set they give requires at most $n=2$, and that only for the transverse rotations which interchange imaginary octonionic units. As they show, these rotations can be generated by two flips, each of which is a pure imaginary multiple of the identity matrix.

Turning to the action of the Lorentz group on spinors $v$, there is another associativity problem. The generators $M$ given by Manogue \& Schray [2] satisfy a compatibility condition between the spinor and vector representations, namely

$$
(M v)(M v)^{\dagger}=M\left(v v^{\dagger}\right) M^{\dagger}
$$

It can be shown that the necessary and sufficient conditions for $M$ to be compatible are that its components all lie in a single complex subspace of the octonions, and that ${ }^{2}$

$$
\operatorname{det} M \in \mathcal{R}
$$

2 This result was checked using Mathematica. 
Note that the determinant is well-defined here because $M$ is complex. ${ }^{3}$

Thus, the (finite) Lorentz transformations are generated by all $2 \times 2$ complex matrices which have determinant \pm 1 . (There is of course no requirement that these matrices all lie in the same complex subspace.) It is interesting to compare this with the complex and quaternionic cases, where compatibility is automatic. In the complex case, multiplication of $M$ by an arbitrary phase $e^{i \theta}$ does not change the vector transformation, but does affect the spinor transformation. The determinant condition (32) eliminates all of these transformations except those for which $\theta=n \pi / 2$. The half-integer multiples of $\pi$ can be eliminated by requiring the determinant to be +1 , leaving only the expected 2 -to- 1 mapping corresponding to an arbitrary overall sign.

The quaternionic case is more subtle: Multiplication by $e^{i \theta}$ now corresponds to a rotation in the $j k$-plane, and therefore must be included as a Lorentz transformation even though its determinant is not real. It is interesting to note that the work of Manogue \& Schray shows how to write such transformations as a product of two flips, each of which has determinant -1 ; the determinant of a product fails to be the product of the determinants in this case. Thus, (32) can indeed be used to define the generators of (finite) Lorentz transformations in this case.

Returning to the octonionic case, all of Manogue \& Schray's generators are complex and either have determinant +1 or are constructed from two nested transformations, each of which has determinant -1 . Thus, the Lorentz group could be defined for each of the division algebras as being generated by such matrices. Furthermore, it is straightforward to write those of Manogue \& Schray's generators with determinant +1 as the product of two transformations with determinant -1 , and it is interesting that, in the octonionic case, the flips themselves can be so generated, even though they have determinant -1 .

We can therefore define the Lorentz group in each case to be those transformations generated by two complex matrices of determinant -1 , which suitably generalizes the more traditional definition in terms of matrices of determinant +1 . It is only in this nested sense that $\operatorname{SL}(2, \mathcal{O})$ consists of "all matrices of determinant +1 ." 4

3 It is intriguing to compare (29) with Dieudonné's prescription [5] for the determinant $\operatorname{Det}(M)$ of a quaternionic matrix $M$, which reduces in the $2 \times 2$ case to (compare [6])

$$
\operatorname{Det}(M)=\sqrt{\operatorname{det}\left(M M^{\dagger}\right)}
$$

where of course $\operatorname{det}\left(M M^{\dagger}\right)$ denotes the ordinary (complex) determinant of $M M^{\dagger}$, which is a positive real number. While the Dieudonné determinant has some nice properties, including the fact that

$$
\operatorname{Det}(M N)=\operatorname{Det}(M) \operatorname{Det}(N)
$$

we choose to work with the ordinary determinant in large part due to the compatibility condition (32), which can not be expressed using the Dieudonné determinant alone, which is always real and positive.

4 There is a notational hazard here: $\operatorname{SL}(2, \mathcal{O})$ could refer to either of two quite different objects, an ambiguity which does not arise over the other division algebras. The first 


\section{OCTONIONIC MÖBIUS TRANSFORMATIONS}

Putting this all together, we will invert the usual derivation that Lorentz transformations are the same as Möbius transformations. Rather, we will define octonionic Möbius transformations in terms of the Lorentz transformations of Manogue \& Schray, and then show that these transformations can be rewritten in the form (17).

Thus, given an octonion $w$, define (generators of) Möbius transformations via (15), which we rewrite as

$$
f_{M}(w)=(\alpha w+\beta)(\gamma w+\delta)^{-1}
$$

and where the matrix of coefficients $M$ defined by (23) is now not only octonionic, but is further required to be one of Manogue \& Schray's compatible generators of the Lorentz group.

We would like to be able to construct more general Möbius transformations by nesting. However, it is not at all obvious that iterating (33) leads to a (suitably nested) transformation of the same type. We would really like to be able to use (an octonionic version of) (17) to define Möbius transformations, as this would make it apparent that iterating Möbius transformations corresponds directly to nesting Lorentz transformations. As previously noted, this requires (17) to be independent of the particular choice of $b$ and c. Remarkably, the octonionic generalization of (17) does have this property, as we now show; this is our main result.

Suppose that

$$
w=b c^{-1}
$$

where now $b, c \in \mathcal{O}$, and construct the spinor $v$ as in (21). Letting

$$
v_{0}=\left(\begin{array}{c}
w \\
1
\end{array}\right)
$$

we have

$$
v=v_{0} c
$$

and

$$
v v^{\dagger}=|c|^{2} v_{0} v_{0}^{\dagger}
$$

since only two octonionic directions are involved.

We now write

$$
V=M v=\left(\begin{array}{l}
B \\
C
\end{array}\right)=\left(\begin{array}{c}
B C^{-1} \\
1
\end{array}\right) C
$$

possibility is the matrix algebra of the $2 \times 2$ matrices just discussed, which is not associative, and hence not a group. The second possibility is the action of these matrices on either spinors (2-component octonionic columns) or vectors $(2 \times 2$ octonionic Hermitian matrices). This is a group; the group operation is composition, which is associative. The main result of [2] is that this group is (isomorphic to) the double cover of $\mathrm{SO}(9,1)$, and hence is isomorphic to $\operatorname{Spin}(9,1)$. It is attractive to write this isomorphism as $\operatorname{SL}(2, \mathcal{O}) \approx \operatorname{Spin}(9,1)$, in which $\mathrm{SL}(2, \mathcal{O})$ refers to the second possibility; we feel that the current paper further supports this usage. However, this notational ambiguity does not affect the statement made in the main text above. 
leading to

$$
V V^{\dagger}=\left(\begin{array}{cc}
|B|^{2} & B \bar{C} \\
C \bar{B} & |C|^{2}
\end{array}\right)=|C|^{2}\left(\begin{array}{cc}
\frac{|B|^{2}}{|C|^{2}} & B C^{-1} \\
\overline{B C^{-1}} & 1
\end{array}\right)
$$

and similar relations for $V_{0}=M v_{0}$. Compatibility now leads to

$$
\begin{aligned}
V V^{\dagger} & =(M v)(M v)^{\dagger} \\
& =M\left(v v^{\dagger}\right) M^{\dagger}=|c|^{2} M\left(v_{0} v_{0}^{\dagger}\right) M^{\dagger} \\
& =|c|^{2}\left(M v_{0}\right)\left(M v_{0}\right)^{\dagger}=|c|^{2} V_{0} V_{0}^{\dagger}
\end{aligned}
$$

Comparing the offdiagonal entries of (40), we obtain

$$
|C|^{2} B C^{-1}=|c|^{2}\left|C_{0}\right|^{2} B_{0} C_{0}^{-1}
$$

But direct computation shows that

$$
|C|^{2}=|\gamma b+\delta c|^{2}=|\gamma w+\delta|^{2}|c|^{2}=\left|C_{0}\right|^{2}|c|^{2}
$$

provided

$$
\langle[b, c, \gamma], \delta\rangle=0
$$

which holds for compatible $M$ since $\gamma$ and $\delta$ lie in the same complex subspace of $\mathcal{O}$. Finally, by construction we have

$$
f_{M}(w)=B_{0} C_{0}^{-1}
$$

and putting this all together results in

$$
B C^{-1}=f_{M}(w)
$$

or equivalently

$$
f_{M}(w)=(\alpha w+\beta)(\gamma w+\delta)^{-1}=(\alpha b+\beta c)(\gamma b+\delta c)^{-1}
$$

This is the desired result, since $b$ and $c$ were arbitrary (satisfying (34)).

\section{PREVIOUS WORK}

Dündarer, Gürsey, \& Tze [3,4] give a Möbius representation of conformal transformations in $\mathcal{R}^{8}$, which relies on a decomposition of the form

$$
G_{2} \subset \operatorname{Spin}(7) \subset \operatorname{Spin}(8) \subset \operatorname{Spin}(9,1)
$$

They thus reduce an arbitrary conformal transformation to a composition of the form $[3,4]$ 5

$$
x \mapsto(U V)^{-1}\left\{V\left(U\left[K\left(L\left(\frac{\lambda}{x-A}+\bar{C}\right)^{-1} \bar{L}\right) K\right] U^{-1}\right) V^{-1}\right\}(U V)
$$

5 The order of $K$ and $L$ can presumably be reversed, although this will change the transformation. 
where the parameters $\lambda \in \mathcal{R}, A, C, K, L \in \mathcal{O}$ with $|K|=1=|L|$ correspond to a dilation, a translation, a special conformal transformation, a rotation in $\operatorname{Spin}(8) / \operatorname{Spin}(7)$, and a rotation in $\operatorname{Spin}(7) / G_{2}$, respectively. They claim the remaining parameters $U, V \in \mathcal{O}$ correspond to a $G_{2}$ transformation, which they give in three forms (page 229 of [4]), related by triality

$$
\begin{aligned}
& y \mapsto(\overline{a b})[b(a y \bar{a}) \bar{b}](a b) \\
& y \mapsto(\overline{a b})\left[b\left(a y a^{2}\right) b^{2}\right](\overline{a b})^{2} \\
& y \mapsto(a b)^{2}\left[\bar{b}^{2}\left(\bar{a}^{2} y \bar{a}\right) \bar{b}\right](a b)
\end{aligned}
$$

where $|a|=1=|b| .{ }^{6}$ Gürsey \& Tze [4] note correctly that if both of $a$ and $b$ admit power series expansions around 1, then the infinitesimal form of each of (49)-(51) agrees with the standard form of $G_{2}$ as the derivation algebra of $\mathcal{O}$ [7]. However, as we now show, the expressions (49)-(51) are not automorphisms for all values of $a$ and $b$; the assumed power series expansions are not valid.

The two octonions $a$ and $b$ span a quaternionic subspace $\mathcal{H} \subset \mathcal{O}$. We can therefore view the octonions as arising from $\mathcal{H}$ via the Cayley-Dickson process, so that

$$
\mathcal{O}=\mathcal{H} \oplus \mathcal{H} e
$$

where $e$ is any pure imaginary octonionic unit orthogonal to $\mathcal{H}$. We can thus write any octonion $x \in \mathcal{O}$ uniquely as

$$
x=x_{1}+x_{2} e
$$

with $x_{i} \in \mathcal{H}$, and the multiplication of two such octonions $x, y$ can be written as

$$
x y=\left(x_{1}+x_{2} e\right)\left(y_{1}+y_{2} e\right)=\left(x_{1} y_{1}-\bar{y}_{2} x_{2}\right)+\left(y_{2} x_{1}+x_{2} \bar{y}_{1}\right) e
$$

Rewriting (49)-(51) in this way, it is lengthy but straightforward to show that (49)-(51) are automorphisms if and only if 7

$$
a b a b a=b a^{3} b
$$

Furthermore, (55) is identically satisfied to second order, thus confirming second-order agreement with the derivation algebra as claimed in [4]. If $a, b$ lie in a complex subspace of $\mathcal{O},(55)$ is trivially satisfied but, using alternativity, each of (49)-(51) reduces to the identity. However, except for this special case, it turns out that a must be purely imaginary. Thus, in no case does an automorphism of the form (49)-(51) admit the assumed power series expansion! 8 We therefore find it remarkable that not only do automorphisms of

6 The expression (48) uses the first form (49) with $a=\frac{U}{|U|}, b=\frac{V}{|V|}$.

7 Interestingly, this is not just an associativity issue: (50)-(51) are not automorphisms of $\mathcal{H}$ unless this condition holds, although (49) is.

8 The first form (49) is also an automorphism if $a b a b a=-b a^{3} b$, in which case both $a$ and $b$ are bounded away from 1 . 
the form (49) exist at all, but enough such automorphisms exist to generate all of $G_{2}$ (by iteration).

However, it is not necessary for the construction of Dündarer, Gürsey, \& Tze that (49) be an automorphism for all values of $a$ and $b$. Rather, it would be enough if every $G_{2}$ transformation could be written in this form. However, this also fails: All of these automorphisms fix precisely 1 octonionic direction (except for special values of the parameters), but there are automorphisms which leave entire quaternionic subspaces invariant. We conclude that the decomposition of Gürsey \& Tze must be modified so as to include (at least) one additional $G_{2}$ transformation.

We now compare this treatment of $G_{2}$ with that of Manogue \& Schray [2], who show how to generate the group $\operatorname{Spin}(7)$ by nesting either conjugation, left multiplication, or right multiplication. Since elements of $G_{2} \subset \operatorname{Spin}(7)$ can be generated by nesting opposite Spin(7) rotations in two planes which "point" towards the same octonionic direction, this yields a nested representation of $G_{2}$. Rewriting these in a form similar to (49)-(51), we obtain

$$
\begin{aligned}
y & \mapsto(d \ell)((c \ell)[d(c y \bar{c}) \bar{d}](\overline{c \ell}))(\overline{d \ell}) \\
y & \mapsto(d \ell)((c \ell)[d(c y)]) \\
y & \mapsto([(y \bar{c}) \bar{d}](\overline{c \ell}))(\overline{d \ell})
\end{aligned}
$$

where $c, d$ are pure imaginary unit octonions and $\ell$ is any imaginary unit octonion orthogonal to the quaternionic subspace spanned by $c, d$. If in addition $c$ and $d$ are orthogonal, then (56)-(58) agree exactly with (49)-(51) under the identification $c=a e, d=b e$, with $e$ orthogonal to $\mathcal{H}$ as before, and where $\ell$ is any (normalized) linear combination of $a$ and $b$. In general, however, (49)-(51) and (56)-(58) give different "bases" for $G_{2}$, since the latter fix a quaternionic subspace and the former do not. In any case, all Möbius representations can also be obtained by iterating (48).

\section{DISCUSSION}

We have shown that the finite octonionic Lorentz transformations in 10 dimensions as given by Manogue \& Schray [2] can be used to define octonionic Möbius transformations, thus recovering (and correcting) the earlier results of Dündarer, Gürsey, \& Tze [3,4]. However, our approach differs significantly from theirs, as theirs corresponds to using (15), while ours uses (17). We have thus shown that octonionic Möbius transformations extend to the octonionic projective space $\mathcal{O} \mathcal{P}^{1}$, defined by ${ }^{9}$

$$
\mathcal{O P}^{1}=\left\{[(b, c)] \in \mathcal{O}^{2}:(b, c) \sim\left(\left(b c^{-1}\right) \xi, \xi\right) \quad \forall 0 \neq \xi \in \mathcal{O}\right\}
$$

We believe that this may be the key result needed to generalize 4-dimensional twistor theory to 10 dimensions. Much recent research in superstrings, supergravity, and M-theory has

9 A related definition in terms of $2 \times 2$ octonionic Hermitian matrices (the "square" of

the form given here) was given by Harvey (page 123 of [8]). However, there is a minor error in his discussion of the equivalence relation, which we have corrected. 
emphasized the importance of lightlike objects in 10 dimensions. An appropriate octonionic generalization of twistor theory to 10 dimensions might allow powerful twistor techniques to be applied to these other theories.

A key role in our argument is the use of two fundamental properties of the octonionic Lorentz transformations in [2], namely nesting and compatibility. Our results here support our view that these are essential features of any computation involving octonions. Otherwise, repeated transformations of the form (15) are not equivalent to those of the form (17), due to the lack of associativity.

Finally, by restricting to a quaternionic subspace of the octonions, we also obtain a corresponding relationship between quaternionic Möbius transformations and the Lorentz group $\mathrm{SO}(5,1)$ in 6 dimensions. Since there are no associativity problems, this could of course have been obtained by straightforward generalization of the complex case.

\section{REFERENCES}

1. Roger Penrose and Wolfgang Rindler, Spinors and Space-Time, Cambridge University Press, Cambridge, 1984 \& 1986, and references cited there.

2. Corinne A. Manogue and Jörg Schray, Finite Lorentz transformations, automorphisms, and division algebras, J. Math. Phys. 34, 3746-3767 (1993).

3. Resit Dündarer, Feza Gürsey and Chia-Hsiung Tze, Self-Duality and Octonionic Analyticity of $S^{7}$-Valued Antisymmetric Fields in Eight Dimensions, Nucl. Phys. B266, 440-450 (1986).

4. Feza Gürsey and Chia-Hsiung Tze, On the Role of Division, Jordan, and Related Algebras in Particle Physics, World Scientific, Singapore, 1996.

5. Emil Artin, Geometric Algebra, John Wiley \& Sons, New York, 1957 \& 1988.

6. E. Study, Acta Math. 42, 1 (1920);

Freeman J. Dyson, Quaternion Determinants, Helv. Phys. Acta 45, 289-302 (1972).

7. Richard D. Schafer, An Introduction to Nonassociative Algebras, Academic Press, New York, 1966 \& Dover, Mineola NY, 1995.

8. F. Reese Harvey, Spinors and Calibrations, Academic Press, Boston, 1990. 